•论坛・

\title{
IPBES框架下的生物多样性和生态系统服务 区域评估及政策经验
}

\section{吴杨 潘玉雪 张博雅 戴逢斌 田 瑜*}

(中国环境科学研究院国家环境保护区域生态过程与功能评估重点实验室, 北京 100012)

\begin{abstract}
摘要: 生物多样性和生态系统服务为人类的生计和良好的生活质量奠定了重要基础。然而, 越来越多的研究表明, 生物多样性和生态系统服务在全球范围内的持续下降使自然对人类的贡献大幅降低。多尺度评估能够说明不同尺 度下生物多样性的现状，有利于制定适合区域特点、符合国情的决策建议。2013年12月，生物多样性和生态系统 服务政府间科学政策平台(Intergovernmental Science-Policy Platform on Biodiversity and Ecosystem Services, IPBES) 通过第一轮工作方案，决定开展“区域/次区域生物多样性和生态系统服务评估”(简称“区域评估”), 即评估亚洲-太 平洋(简称亚太)、美洲、非洲以及欧洲-中亚四大地理区域的生物多样性和生态系统服务。区域评估报告及其决策 者摘要已在IPBES第六次全体会议上(2018年3月, 哥伦比亚麦德林)审议通过。本文概述了四大地理区域的生物多 样性的重要性、生物多样性保护领域取得的进展、面临的主要危机和机遇, 探讨了评估对其他国际进程的影响, 综 合分析了各区域生物多样性和生态系统服务的特点以及各区域评估结果的差别, 总结了评估的政策经验, 以期为 中国的生物多样性保护提供科学参考。
\end{abstract}

关键词: IPBES; 区域; 生物多样性; 生态系统服务; 评估

\section{Regional assessment on biodiversity and ecosystem services and policy experience within the IPBES framework}

\begin{abstract}
Yang Wu, Yuxue Pan, Boya Zhang, Fengbin Dai, Yu Tian*
State Environment Protection Key Laboratory of Regional Eco-process and Function Assessment, Chinese Research Academy of Environmental Sciences, Beijing 100012

Abstract: Biodiversity and ecosystem services have laid important foundations for human livelihoods that impact quality of life. However, recent studies have shown a continuous decline globally, thereby reducing human interaction with nature. Multi-scale assessments can explain the current status of biodiversity at different scales which can help formulate decision-making recommendations for regional and national agencies. In December 2013, the Intergovernmental Science-Policy Platform on Biodiversity and Ecosystem Services (IPBES) adopted the first work programme and decided to undertake "regional/sub-regional assessment on biodiversity and ecosystem services” (regional assessment). Biodiversity and ecosystem services in the four geographic regions of Asia-Pacific, the Americas, Africa and Europe-Central Asia were assessed. The four regional assessment reports and their summary for policymakers were adopted at the 6th Session of the IPBES Plenary (held March 2018 in Medellin, Colombia). This paper summarizes the importance of biodiversity in each of the four geographic regions and the progress made in biodiversity conservation, the major crises and opportunities, and explores the impact of the assessment on other international processes. This research analyzes the characteristics of biodiversity and ecosystem services in each region and the differences between regions, along with a summary of the policy experience of the assessment to provide important scientific reference for China's biodiversity conservation.
\end{abstract}

Key words: IPBES; regional; biodiversity; ecosystem services; assessment

收稿日期: 2019-10-24; 接受日期: 2020-02-22

基金项目: 生态环境部生物多样性保护专项(22110404001)

* 通讯作者 Author for correspondence. E-mail: tianyu@craes.org.cn 
生物多样性和生态系统服务是人类生计和良 好生活质量的基础。然而, 近年来, 由于人类活动 不断加剧, 生物多样性正以前所未有的速度丧失 (马克平, 2016), 自然对人类的贡献(如能源、传粉、 气候调节)大幅降低, 全球有 100 万个物种可能面临 灭绝的风险(IPBES, 2019)。随着国际社会对生物多 样性保护和人类福祉关注度的不断提高, 评估人类 活动对生物多样性的影响, 预测生物多样性丧失对 人类福祉的影响及保护应对措施，成为当前国际社 会、各国政府和科学界普遍关注的热点问题(Butchart et al, 2010)。生物多样性和生态系统服务政府 间科学政策平台(Intergovernmental Science-Policy Platform on Biodiversity and Ecosystem Services, IPBES)于2012年4月在巴拿马宣布正式成立, 现有 136个成员国。作为生物多样性领域第一个独立的 政府间机制, IPBES旨在通过加强科学政策对生物 多样性和生态系统服务的影响, 从而实现生物多样 性保护与可持续利用, 以及人类的长期福祉与可持 续发展。在中国、挪威、联合国环境规划署、泛欧 平台、世界自然保护联盟(IUCN)及《生物多样性公 约》等国家、机构和组织的积极影响下, IPBES在其 第二次全体会议上决定，在区域尺度上对有关生物 多样性和生态系统服务的知识状况及其相互联系 开展评估。2014年, IPBES启动了四大地理区域评估 工作。2018年3月, 四大地理区域关于生物多样性和 生态系统服务的四份评估报告的决策者摘要在第 六次全体会议上正式发布, 评估报告由来自全球 100多个国家的550多位专家共同撰写(张博雅等, 2018)。

IPBES区域评估报告是具有里程碑意义的科学 报告, 评估准则为全球评估范围界定和工具等提供 了科学依据(田瑜等, 2015)。评估的成果可能对各区 域的经济发展、生计、粮食安全、自然贡献和人类 福祉等领域产生重要影响, 从而有利于推动各区域 为实现联合国可持续发展目标做出贡献。此外, 评 估可对相关公约未来决策和履约走向发挥重要引 导作用。评估报告将引领生物多样性和生态系统服 务相关科学领域的发展, 为各国在生物多样性领域 进行对话、权衡与博弯, 实现本国利益最大化提供 一个高效的平台。

本文在IPBES概念框架基础上, 通过对四个区 域评估报告的概述和分析, 结合各区域特点探讨各
区域生物多样性和生态系统服务的状况及差别, 并 为了解各区域立场提供有力支撑, 为未来履约谈判 提供科学建议。

\section{评估内容和结果}

依据生物地理特征、地理邻近性、生态和气候 相似性、陆地和水生生态系统及生态特征相似性等 原则, IPBES将全球范围分为四大地理区域, 即亚 太区域、美洲区域、非洲区域、欧洲和中亚区域。 国家管辖范围以外的海域不单独做评估, 将涵盖在 全球评估中(田瑜等, 2015)。IPBES区域评估就是对 这四大地理区域范围内的生物多样性和生态系统 服务开展评估, 具体包括: 生物多样性和生态系统 服务的最新状况和趋势; 生物多样性和生态系统服 务的丧失对人类福祉的潜在影响, 以及改变生态系 统的直接和间接驱动因素; 有哪些政策和措施有利 于从生物多样性和生态系统服务的角度提高人类 福祉及其有效性。

\section{1 亚太区域评估}

亚太区域分布的国家和领土最多，社会文化多 元化, 地方和土著社区密度最大(IPBES，2015)。全 球约 $90 \%$ 的水产养殖位于亚太区域，因此该区域人 民严重依赖渔业产品。丰富的生物多样性和生态系 统服务为 45 亿居民提供了粮食、水、能源、文化和 精神支撑, 为亚太地区的经济增长作出了重要贡献, 使该区域成为城市化速度最快的区域之一(IPBES, 2018a)。然而, 高速经济增长和快速城市化导致了 生物多样性加剧退化和丧失。除东北亚和南亚一些 地区的状况维持较好之外, 多数区域的生物多样性 和生态系统状况正在恶化。我国位于东北亚地区, 由于实行了生态系统服务付费、退耕还林、保护区 管理及脱贫等政策, 该地区1990-2015年森林覆盖 率增加了 $22.9 \%$, 保护区的覆盖率也大幅增加, 是 亚太区域贫困率(1.8\%)最低的次区域。

要实现粮食、水和能源安全的可持续发展目标, 亚太区域国家需考虑将生物多样性纳入国家发展 政策和计划，构建综合管理框架，并制定可持续生 产、消费和废物管理政策。亚太区域评估进程将与 《生物多样性公约》《濒危野生动植物种国际贸易 公约》等多边环境协定的区域特定活动以及太平洋 区域环境方案等区域机构建立起密切联系, 为实现 《爱知生物多样性目标》、联合国可持续发展目标 
(2015-2030年)以及《巴黎协定》目标提供建议及参 考案例, 例如在森林及保护区管理方面的进展 (IPBES, 2018b)。

\section{2 美洲区域评估}

美洲是一个生物多样性极高的区域，拥有 $20 \%$ 的关键生物多样性区域和 $26 \%$ 的陆地生物多样性热 点区域。陆地生态系统每年至少带来24.3万亿美元 的经济价值，为美洲发展经济、保障民生、消除贫 困和提高生活质量做出了重要贡献。此外, 美洲土 著人民和地方拥有丰富的文化, 提供大量的土著和 地方知识来管理生物多样性和自然对人类的贡献 (IPBES, 2015)。美洲作为全球最大的粮食出口地和 最大的生物能源交易地，其人均资源量是世界平均 水平的3倍(IPBES, 2018c)。美洲淡水资源丰富，但 由于水质下降，人均淡水占有量逐年下降。此外, 美洲横跨南北极, 次区域的社会、经济和生态状况 差异较大, 存在资源分配不均的现象, 特别是中美 洲、加勒比和南美洲地区仍存在不少贫困人口和弱 势群体。

评估结果表明, 虽然一些退化的生境因采取恢 复措施已得到改善, 但是美洲的大部分生态系统仍 在退化。例如虽然加勒比地区的森林覆盖率提高, 然而已恢复的地区仍只占美洲区域很小一部分。生 物多样性持续丧失阻碍了可持续发展目标及与气 候相关的目标的实现，该区域大多数国家似乎难以 在2020年这一最后期限前实现大部分的爱知目标 (IPBES, 2018d)。

\section{3 非洲区域评估}

丰富的生物多样性和生态系统服务以及土著 和地方知识财富是非洲区域可持续发展的战略资 源。非洲是地球上最后一个拥有众多大型哺乳动物 的区域, 拥有沙漠、高山、海洋和热带雨林等丰富 多样的生态系统, 为非洲人民提供了粮食、水、能 源等至关重要的供给服务, 该区域人民的生计需求 高度依赖于生态系统服务。在过去20年中, 非洲经 济得到了发展，但依旧摆脱不了极端贫困的困境， 粮食、水和能源得不到保障, 严重影响到非洲人民 的生计(IPBES, 2015), 导致社会关系紧张, 引起人 与人的不平等, 这种不平等是非洲大陆许多冲突的 根源(IPBES, 2018e)。

非洲国家正在制定生物多样性战略和行动计 划, 虽然在履行《2011-2020年全球生物多样性战略
计划》的承诺方面取得了一些进展，但是由于资金 和能力建设等限制, 许多非洲国家的行动进展仍然 缓慢, 为实现《爱知生物多样性目标》做出的贡献 较少，非洲联盟难以如期完成《2063年议程》目标 (IPBES, 2018e)。因此，非洲须充分发挥该区域生物 多样性的价值，采取可持续和公平的利用方式，以 减少非洲大陆生物多样性丧失、不平等和贫穷的现 象(IPBES, 2018f)。

\section{4 欧洲和中亚区域评估}

欧洲和中亚区域的生物多样性独一无二，具有 重大的经济、社会和文化价值。自然为人类提供食 物和药材, 并提供绿色空间保障人类身心健康。土 著人民和地方社区的知识和习俗可以保护文化遗 产，从而提高人们的生活质量。调节服务的价值最 高，其中调节淡水质量的价值每年高达 1,965 美元 /ha。然而, 欧洲和中亚区域的生物多样性正在持续 丧失，许多调节作用(土壤形成、传粉和淡水质量调 节)大幅下降(IPBES, 2018g)，欧盟25\%、中亚 23\%的 农业用地的土壤受到侵蚀, 影响了粮食生产; 中亚 大约有 $15 \%$ 的居民无法获得安全的饮用水。淡水物 种和内陆地表水生境受到的威胁尤为严重，71\%的 鱼类和 $60 \%$ 的两栖动物的数量在过去 10 年持续减少, 湖泊和溪流逐渐消失。部分地区的生物承载力不再 能够承受居民的生态足迹，尤其是中欧和西欧地区， 因此这些地区的粮食和木材等不得不依赖进口，大 批量的进口对世界其他地区造成了一些不利影响, 比如全球年度森林砍伐量中有 $10 \%$ 是欧盟 27 个成员 国的消费造成的。

近年来，欧洲和中亚采取的可持续发展和保护 行动有助于扭转生物多样性减少的趋势。就实现可 持续发展目标而言，欧洲和中亚(特别是欧洲)在环 境保护、人类健康、粮食安全和水安全方面总体上 取得了一些积极的进展，渔业的可持续管理使得西 欧北海的鱼群数量增加, 马卡罗尼西亚林地等生境 已大幅恢复。继续加大保护力度并采取更积极、有 针对性的治理方式, 将有效促进生物多样性目标的 实现(IPBES, 2018h)。

\section{四大区域评估结果对比分析}

四大区域生物多样性的评估结果基于数千篇 科学文献和报告以及土著和地方知识。尽管在四个 区域有许多共同的发现，但由于社会、文化、经济、 
地理环境等方面的特性不同, 各区域在自然对人类 的贡献、生物多样性丧失的驱动因素以及面临的挑 战和威胁等方面仍存在差异。

\section{1 自然对人类的贡献}

在区域评估报告中, IPBES首次提出了自然对 人类的贡献(Nature's Contributions to People, NCP) 这一概念, 旨在更全面地考虑自然与人类的关系。 自然对人类共有18种贡献(IPBES, 2019), 不仅涵盖 了自然对人类生活质量的积极贡献，还包括了自然 对人类的消极贡献(例如疾病和灾害), 其中许多贡 献和生态系统服务的分类密切对应。NCP对于维持 良好的生活质量至关重要。自然为各区域的居民提 供了调节贡献(传粉、气候调节和淡水调节等)、物 质贡献(食物、水和能源等)和非物质贡献(学习和体 验等)。受地理环境和社会经济差异的影响, 自然为 各区域提供的最重要的贡献有所不同。除了满足居 民的生计需求, 亚太区域的生物多样性和生态系统 还满足居民的文化和精神需求; 美洲区域的生态系 统在调节服务和文化服务方面做出了重要贡献; 欧 洲和中亚区域的生态系统的调节贡献具有很高的 经济价值, 例如调节淡水(1,965美元/ha·年)、生境维 护(765美元/ha·年)、调节气候(464美元/ha·年)、调节 空气质量(289美元/ha·年)等; 非洲区域最重要的 NCP就是食物、能源、医疗资源等物质贡献, 因此 居民生计高度依赖大自然。然而, 由于生物多样性 丧失的状况不断加剧, 各区域NCP普遍呈现下降趋 势，将对居民的生活质量造成消极影响。

\section{2 生物多样性丧失的驱动因素}

在众多因素的驱动下, 四大区域的生物多样性 和生态系统服务都受到了较为严重的影响(图1)。人 口增长、经济增长、快速城市化是各区域生物多样 性丧失最重要的间接驱动因素, 人类对自然资源的 需求增加, 导致土地用途改变, 对自然造成了极大 的破坏。气候变化、外来物种入侵、资源过度利用、 农业集约化、污染等是最主要的直接驱动因素。气 候变化影响气温和降水, 将引起冰川融化、洪水、 旱灾、疾病传播等一列极端事件, 今后可能成为全 球面临的最紧迫的挑战。因此, 全球各国都有责任 和义务植树造林、减少温室气体排放, 为缓解气候 变化做出努力, 协助国际社会观测和预测气候变化 的影响, 助力实现《巴黎协定》的目标。同时, 外 来入侵物种的数量和丰度增加, 也是造成各区域生
物多样性减少的重要因素, 需要加强监督和管理。

然而, 对各区域来说, 生物多样性丧失的关键 驱动因素各有不同。对亚太区域来讲，外来物种入 侵和资源不可持续利用是造成生物多样性减少最 重要的原因，亚太区域的许多国家仍然面临长期贫 困，正通过扩大贸易来谋求经济快速发展，增加了 外来物种入侵的风险。土地用途改变和气候变化是 美洲区域以及欧洲和中亚区域生物多样性减少的 最主要的驱动因素, 为了提高粮食和生物燃料的生 产，这些区域采用了集约式农业和林业，进而引发 生境退化、湿地退化、城市化和资源开采，对生物 多样性造成了巨大的威胁。在未来，气候变化和其 他驱动因素共同作用，破坏力将非常大。非洲区域 也极易受气候变化的影响, 所有非洲国家的气温上 升速度预计高于全球平均速度，造成非洲动植物物 种(高达 $50 \%$ 以上)严重损失、湖泊生产力下降以及 海洋生态系统破坏。

\section{3 威胁和挑战}

受不同驱动因素的影响，各区域面临的威胁和 挑战具有区域差异性。亚太区域国家快速城市化以 及人口增多使得资源消耗问题加重，进而引发了粮 食、水、能源的安全问题，因此亚太区域非常重视 可持续发展; 亚太区域塑料废物占全球海洋废物总 量的 $95 \%$ ，全球塑料废物最多的 10 条河流中有 8 条 在亚洲，水源和空气污染给人类健康造成了持久的 威胁，废物管理也成为一项重大挑战; 珊瑚礁和海 洋生态系统面临严重的威胁, 须采取跨界区域合作 的方式对严重濒危的生物景观、文化景观和海洋景 观进行保护。美洲区域同样存在快速城市化的问题, 造成了严重的环境污染和土地退化。虽然美洲淡水 资源丰富，但是由于淡水资源分配不均和水质下降， 使得美洲有一半以上的居民用水得不到保障, 因此 美洲区域须重视资源公平分配的问题。欧洲和中亚 区域长期面临大量可再生资源依赖进口的问题，因 此该区域十分注重可持续生产和节约资源，欧洲和 中亚区域拥有丰富的政策经验和先进的生态技术 (例如自然资本核算工具), 有利于解决该区域生态 和经济权衡取舍的问题，但是由于资源生产力的明 显限制，该区域的问题并不能在短时期内得到有效 解决。非洲人民高度依赖自然，该区域的生计问题 显得尤为严峻，可持续利用农业生物多样性将有效 解决该区域粮食短缺的难题，因此较其他区域来 


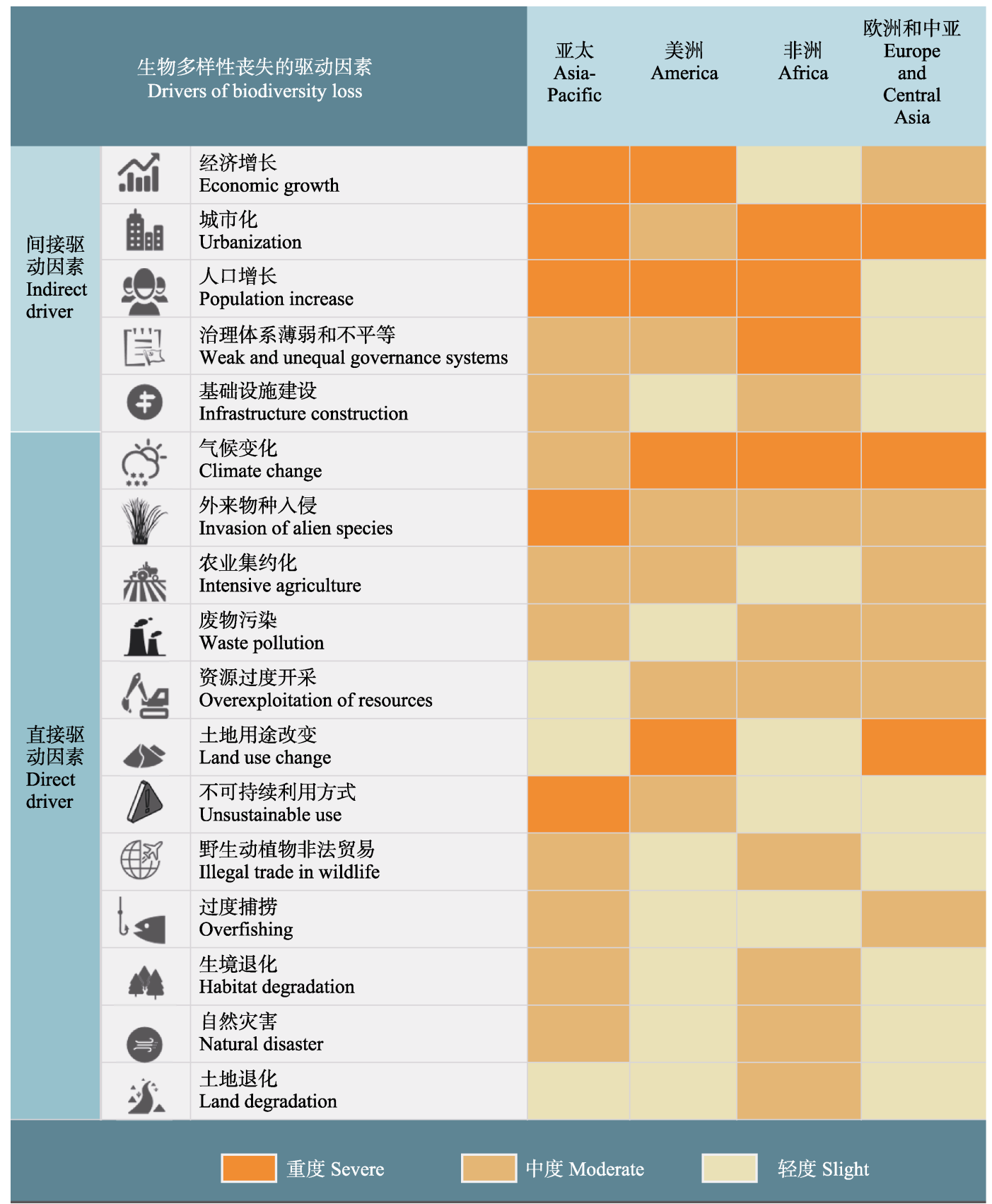

图1 四大区域生物多样性丧失的驱动因素分析

Fig. 1 Analysis of the driving factors of biodiversity loss in four regions

说，非洲区域面临的问题和挑战相对简单。

\section{3 政策经验}

\section{1 推进生物多样性主流化}

《生物多样性公约》第十三次缔约方大会上提 出 “为实现人类福祉, 推动生物多样性主流化”, 并 在墨西哥坎昆高级别会议上通过了《将保护和可持 续利用生物多样性纳入主流化以增进福祉的坎昆
宣言》。把生物多样性纳入到国家或地方政府的政 治、经济、社会、军事、文化及环境保护等经济社 会发展建设的主流，将极大地推动区域实现可持续 发展。从各区域的政策选择来看, 均反映了对生物 多样性主流化的迫切需求: 亚太区域和非洲区域需 要将保护和可持续利用生物多样性纳入到金融、农 业、能源和水资源等部门和机构的决策进程中; 美 洲区域以及欧洲和中亚区域采用生态系统服务付 
费、自愿性生态认证等环境政策工具调动企业和社 会的积极性(IPBES, 2018d)。中国政府首次将生物多 样性和生态系统服务价值纳入政绩考核和干部离 任审计, 重庆市、安徽省安庆市和云南省景东县等 地已经开始实行。该政策从根本上解决了当地经济 发展与生物多样性保护的矛盾, 下一步可考虑在全 国范围推进实施。

\section{2 鼓励多方参与}

加强多方参与, 有助于在政策制定和决策中整 合不同形式的知识, 促进责任分担。各区域均意识 到多方参与的重要性。亚太区域重视与私营部门、 个人和非政府组织建立伙伴关系, 为各项保护工作 提供了资金保障(IPBES，2018b); 欧洲和中亚区域 将环境治理的决策权逐步下放给私人管理，提高了 环境治理的成效和公平性; 非洲区域及美洲很多地 区强调要确保土著人民、地方社区及弱势群体的权 利, 增加把土著和地方知识纳入生态系统管理和决 策的机会。近年来, 我国一些企业积极参与生物多 样性保护尝试, 阿里巴巴与国际野生物贸易研究组 织(TRAFFIC)合作，遏制网络非法野生物贸易; 社 区和公众参与也是我国生物多样性保护的关键力 量, 如三江源国家公园在社区牧民中招募公益巡护 员，实现了共治和共享。

\section{3 加强国际合作与交流}

国际合作是促进全球生物多样性保护的必要 手段。生物多样性面临的很多威胁(例如海洋酸化、 海洋塑料污染、气候变化以及野生动植物的非法贸 易等)实际上是跨国界的, 可以通过多边协定和国 际合作来有效解决。亚太区域在海洋景观跨界保护 过程中鼓励多边签订《关于珊瑚礁、渔业和粮食安 全的珊瑚三角区倡议》和《冈仁波齐神山圣地景观 保护和开发倡议》, 推动了海洋景观的协作管理 (IPBES, 2018b); 非洲区域在保护淡水和海洋系统 及动物迁徙的生态系统时开展了次区域合作。近年 来, 我国不断推动多边、双边和南南合作, 深化国 际合作与交流，加强在 “一带一路”倡议下支撑和保 障全球生物多样性的保护和研究, 推进生物多样性 履约与国际合作, 在国际合作和交流方面取得了显 著成效。

\section{4 推动转型变革}

IPBES指出, 若要实现2030年或者更长期的自 然保护目标, 转型变革势在必行(IPBES, 2019)。亚
太区域在社会经济快速发展过程中付出了巨大的 环境代价, 如果按照现有的保护模式, 生物多样性 保护和可持续发展目标将无法实现，引入与社会经 济体制匹配的转型方案和政策(例如绿色增长和低 碳), 有利于解决这一问题; 美洲区域希望通过消费 转型减轻对自然界的压力; 欧洲和中亚区域提出通 过不断开展教育、知识共享和参与式决策实现长期 社会转型; 非洲联盟《2063年议程》旨在推动必要 的变革、发展和转型, 实现大幅度减贫。中国的生 物多样性保护形势依旧严峻, 需要通过法律、行政、 市场、技术、社会等五方面推动变革(杨锐等, 2019), 例如, 进一步完善生物多样性保护的法律法规体系 建设, 加强人才培养和科学研究, 注重生态资本核 算，建立生态系统服务的交易市场; 立足脚下，从 小事出发, 可以是价值观和饮食习惯的转变, 也可 以是资源利用方式和垃圾分类方式的转变, 只有将 生物多样性保护真正与每个公民的切身利益挂钩, 凝聚全民力量才能实现真正的变革。

\section{问题与展望}

区域评估取得了丰富的成果和经验，然而，在 评估过程中也发现了一些不足之处。(1) IPBES评估 是对现有科学知识进行分析整理, 形成有价值的评 估报告，而IPBES 目前正在起步阶段，评估的工具 和方法有待完善。(2)评估的关键信息和数据存在空 缺, 甚至存在严重的知识空缺, 例如自然对人类的 贡献多重价值有待进一步量化和综合监测，对土著 和地方知识的贡献了解不足，缺乏综合性情景模拟 研究等。如果今后的评估要更全面地阐述自然及其 对人类贡献的状态和趋势, 就需要填补攻克这些知 识空缺。(3)评估在一定程度上暴露出资金和能力空 缺，资金缺乏和机构能力不足是保护生物多样性的 重要制约因素，对非洲区域的影响最大，而且评估 不涉及国家尺度, 各个国家需要单独开展国内评估, 这对于经济技术发展落后的国家来说存在一定困 难。因此未来评估需加大资金投入以支持研究、培 训、能力建设、教育和监测活动。

生物多样性丧失属于环境问题，同时也映射出 道德、社会和经济发展的问题。当前各国政府、私 营部门和公众的决定和行动将直接影响后代的生 存环境质量。2020年是《2011-2020生物多样性战 略计划》实施的收官之年, 但爱知目标完成情况并 
不理想，全球生物多样性丧失问题依旧严峻，全球 的生物多样性保护需要得到高度重视, 转型变革不 失为一种很好的解决途径。当务之急须考虑为全球 下一阶段的生物多样性保护制定出有效的、具有指 导性的战略目标及行动计划。

\section{参考文献}

Butchart SHM, Walpole M, Collen B, Strien A, Scharlemann JPW, Almond REA, Baillie JEM, Bomhard B, Brown C, Bruno J, Carpenter KE, Carr GM, Chanson J, Chenery AM, Csirke J, Davidson NC, Dentener F, Foster M, Galli1 A, Galloway JN, Genovesi P, Gregory RD, Hockings M, Kapos V, Lamarque JF, Leverington F, Loh J, McGeoch MA, McRae L, Minasyan A, Morcillo MH, Oldfield TEE, Pauly D, Quader S, Revenga C, Sauer JR, Skolnik B, Spear D, Smith DS, Stuart SN, Symes A, Tierney M, Tyrrell TD, Vié JC, Watson R (2010) Global biodiversity: Indicators of recent declines. Science, 328, 1164-1168.

IPBES (2015) Report on the Regional Scoping Process for a Set of Regional and Subregional Assessments (IPBES/3/6). https://www.ipbes.net/event/ipbes-3-plenary. (accessed on 2019-08-06)

IPBES (2018a) The IPBES Regional Assessment Report on Biodiversity and Ecosystem Services for Asia and the Pacific. Bonn, Germany. https://ipbes.net/assessment-reports /asia-pacific. (accessed on 2019-08-06)

IPBES (2018b) Summary for Policymakers of the Regional Assessment Report on Biodiversity and Ecosystem Services for Asia and the Pacific of the Intergovernmental Science-Policy Platform on Biodiversity and Ecosystem Services. Bonn, Germany. https://ipbes.net/assessment-repor ts/asia-pacific. (accessed on 2019-08-06)

IPBES (2018c) The IPBES Regional Assessment Report on Biodiversity and Ecosystem Services for the Americas. Bonn, Germany. https://ipbes.net/assessment-reports/americas. (accessed on 2019-08-06)

IPBES (2018d) Summary for Policymakers of the Regional Assessment Report on Biodiversity and Ecosystem Services for Americas of the Intergovernmental Science-Policy Platform on Biodiversity and Ecosystem Services. Bonn, Germany. https://ipbes.net/assessment-reports/americas. (accessed on 2019-08-06)

IPBES (2018e) The IPBES Regional Assessment Report on Biodiversity and Ecosystem Services for Africa. Bonn, Germany. https://ipbes.net/assessment-reports/africa. (accessed on 2019-08-06)

IPBES (2018f) Summary for Policymakers of the Regional
Assessment Report on Biodiversity and Ecosystem Services for Africa of the Intergovernmental Science-Policy Platform on Biodiversity and Ecosystem Services. Bonn, Germany. https://ipbes.net/assessment-reports/africa. (accessed on 2019-08-06)

IPBES (2018g) The IPBES Regional Assessment Report on Biodiversity and Ecosystem Services for Europe and Central Asia. Bonn, Germany. https://ipbes.net/assessment-reports/eca. (accessed on 2019-08-06)

IPBES (2018h) Summary for Policymakers of the Regional Assessment Report on Biodiversity and Ecosystem Services for Europe and Central Asia of the Intergovernmental Science-Policy Platform on Biodiversity and Ecosystem Services. Bonn, Germany. https://ipbes.net/assessment-repor ts/eca. (accessed on 2019-08-06)

IPBES (2019) Summary for Policymakers of the Global Assessment Report on Biodiversity and Ecosystem Services of the Intergovernmental Science-Policy Platform on Biodiversity and Ecosystem Services. Bonn, Germany. https://ipbes.net/global-assessment-report-biodiversity-ecosy stem-services. (accessed on 2019-10-08)

Ma KP (2016) Hot issues in biodiversity science. Biodiversity Science, 24, 1-2. (in Chinese) [马克平 (2016) 生物多样性 科学的热点问题. 生物多样性, 24, 1-2.]

Tian Y, Li JS, Lan CZ, Li XS (2015) Interpretation of the work programme of Intergovernmental Science-Policy Platform on Biodiversity and Ecosystem Services for the period 2014-2018. Biodiversity Science, 23, 543-549. (in Chinese with English abstract) [田瑜, 李俊生, 兰存子, 李秀山 (2015) 生物多样性和生态系统服务政府间科学-政策平 台2014-2018年工作方案解析. 生物多样性, 23, 543-549.]

Yang R, Peng QY, Cao Y, Zhong L, Hou SY, Zhao ZC, Huang C (2019) Transformative changes and paths toward biodiversity conservation in China. Biodiversity Science, 27, 1032-1040. (in Chinese with English abstract) [杨锐, 彭钦 一, 曹越, 钟乐, 侯姝或, 赵智聪, 黄澄 (2019) 中国生 物多样性保护的变革性转变及路径. 生物多样性, 27 , 1032-1040.]

Zhang BY, Pan YX, Xu J, Tian Y (2018) The sixth plenary meeting of the Intergovernmental Science-Policy Platform on Biodiversity and Ecosystem Services was held in Colombia. Biodiversity Science, 26, 282. (in Chinese) [张博雅, 潘玉雪, 徐靖, 田瑜 (2018)生物多样性和生态系统服务 政府间科学政策平台第六次全体会议在哥伦比亚召开. 生物多样性, 26, 282.]

(责任编委: 薛达元 责任编辑: 时意专) 